\title{
Hydrocarbon transport in the MkIIa divertor of JET
}

\author{
A Kirschner ${ }^{1}$, J N Brooks ${ }^{2}$, V Philipps ${ }^{1}, \mathrm{~J} \mathrm{P} \mathrm{Coad}^{3}$ and contributors to \\ the EFDA-JET Workprogramme ${ }^{4}$
}

\author{
${ }^{1}$ Institut für Plasmaphysik, Forschungszentrum Jülich GmbH, EURATOM Association, \\ Trilateral Euregio Cluster, D-52425 Jülich, Germany \\ 2 Argonne National Laboratory, Argonne, IL 60439, USA \\ ${ }^{3}$ EURATOM/UKAEA Fusion Association, Culham Science Centre, Abingdon, \\ Oxon OX14 3DB, UK
}

Received 20 May 2002, in final form 23 January 2003

Published 24 February 2003

Online at stacks.iop.org/PPCF/45/309

\begin{abstract}
This contribution presents two-dimensional Monte-Carlo simulations of the local transport of hydrocarbons that are chemically eroded in the JET MkIIa divertor. The effect of a given background carbon flux flowing from the main plasma down to the divertor is also taken into account. The influence of local plasma temperatures and densities and the influence of different assumptions for the sticking of re-deposited particles hitting the tiles is analysed. Even under the assumption that the sticking of re-deposited hydrocarbon fragments is zero, a large amount (about 75\%) of the eroded particles is re-deposited in the form of ionized carbon on the tiles. A reasonable agreement between the simulation and the experimental observation of the carbon deposition at the inner louvers in the MkIIa JET divertor is achieved, if high chemical erosion yields of about $20 \%$ together with a negligible effective sticking of hydrocarbons are assumed. Although there are experimental observations indicating such high erosion yields, such yields cannot be applied as a stationary value for the effective erosion since it would turn the inner divertor into a net erosion area in contrast to the experimental findings. The possibility of applying different erosion rates for (re)-deposited layers and the substrate material is discussed. To further improve the modelling a better knowledge about the formation and erosion of re-deposited layers is required. A first conclusion concerning the erosion and re-deposition behaviour in ITER will be drawn.
\end{abstract}

\section{Introduction}

In fusion research carbon-based materials are still a frequent choice for plasma facing elements exposed to the high heat and particle fluxes near the strike zone areas in the lower divertor area. Thus the vertical targets in the divertor of ITER [1] will be CFC to handle the stationary heat fluxes (up to $20 \mathrm{MW} \mathrm{m}^{-2}$ ) but in particular the transient power pulses in ELMs or disruptions

4 See annex of J Pamela et al 2001 Overview of recent JET results and future perspectives Proc. 18th Int. Conf. on Fusion Energy 2000 (Sorrento, 2000) (Vienna: IAEA). 
[2]. The understanding of the erosion and (re)-deposition behaviour of carbon-based materials, especially under the low temperature plasma conditions that are typical for the divertor regions is of vital importance. The uncertainties about the physical processes involved and the experimental database are still too large for reliable predictions of these processes for future devices. Chemical erosion is the dominant erosion process at the low electron temperatures in the divertor area. A thorough modelling of the carbon transport and comparison with experimental results in existing devices is one of the most important issues in present PSI research. Based on a reasonable reproduction of various experimental results under different conditions in existing devices, the lifetime of the divertor targets and the tritium retention due to co-deposition of eroded hydrocarbons should be predicted for future machines like ITER.

This paper presents detailed modelling studies of the carbon transport in the divertor of JET. In the MkIIa divertor of JET [3] a strong asymmetry of carbon deposition between inner and outer divertor is observed showing that the inner divertor is everywhere deposition dominatedwith most of the deposition on the inner louvers-but very little deposition occurs in the outer divertor including the louver region [4-6]. The inner and outer louvers are indicated in figure 1. The thick carbon deposits on the inner louvers contain the majority of the hydrogenic species retained on a long-term basis. The in-out asymmetry of the particle fluxes is explained with flows in the SOL which drive particles from the SOL in the main chamber towards the inner divertor [4]. This is concluded from measurements of the Mach number [7] but also supported from the fact that beryllium which is evaporated in the main chamber but not in the divertor region, is found only on the inner divertor tiles. Recently, moreover, ${ }^{13} \mathrm{C}$ marked methane has been injected from the top of the machine and also was found only in the inner divertor [8]. The nature of these strong flows is not fully understood. Equally, the local transport of carbon inside the divertor region with the large carbon deposition at the remote, cold locations of the inner water-cooled louvers is not yet understood. Attempts to model this behaviour have been performed with an adapted version of the ERO-TEXTOR code (ERO-JET) [9]. The transport of carbon towards the inner louvers is analysed applying various parameter variations such as electron temperature and density, location of the strike point and sticking assumptions for carbon ions and hydrocarbon fragments. Carbon is released from the target in the form of methane and higher hydrocarbons by chemical erosion but in addition a certain amount of carbon flowing with the background plasma to the divertor surfaces is taken into account.

Starting-point for the simulations are the divertor plasma parameters of a 'representative' standard gas fuelled ELMy H-mode discharge (no 44029, $12 \mathrm{MW}$ heating power) of MkIIa. The density and temperature distributions are calculated from the Onion Skin Model [10-12] using target Langmuir data. They are shown in figure 1. The areas for which the temperature and density is plotted correspond to the calculation volume used for the ERO-JET simulations. These parameters represent a condition where the inner divertor plasma is partially detached whereas at the outer plate an attached plasma is formed with electron temperatures $\left(T_{\mathrm{e}}\right)$ of about $6 \mathrm{eV}$ at the inner strike point and $10 \mathrm{eV}$ at the outer strike point and densities $\left(n_{\mathrm{e}}\right)$ of about $6 \times 10^{13} \mathrm{~cm}^{-3}$ and $6.5 \times 10^{13} \mathrm{~cm}^{-3}$, respectively. From the $T_{\mathrm{e}}$ and $n_{\mathrm{e}}$ profiles along the plates the incoming parallel deuterium ion flux is calculated according to $\Gamma^{\mathrm{D}+}=n_{\mathrm{e}} c_{\mathrm{s}}$ with $c_{\mathrm{s}}=c_{\mathrm{s}}\left(T_{\mathrm{e}}\right)$ the ion flow velocity.

\section{Modelling results of the local carbon transport in MkIIa}

\subsection{Chemically eroded methane $C D_{4}$}

To start with, the incoming deuterium ion flux is assumed to erode methane $\mathrm{CD}_{4}$ with a fixed erosion yield of $1 \%$. This number will be discussed later. To investigate the influence of 

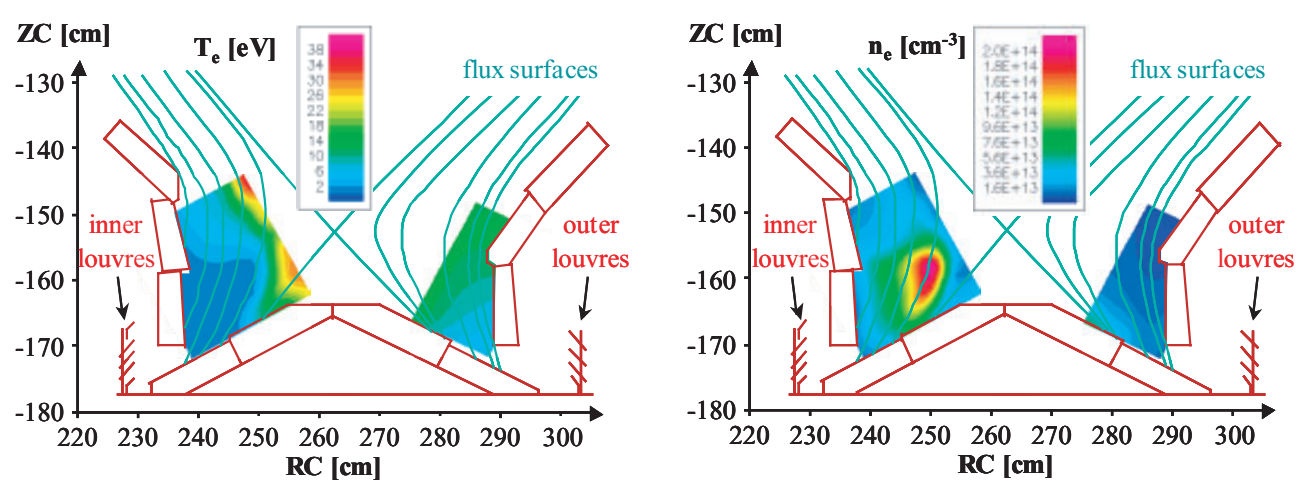

Figure 1. Electron density and temperature distribution calculated from the Onion Skin Model for a representative ELMy H discharge in JET MkIIa (no 44029).
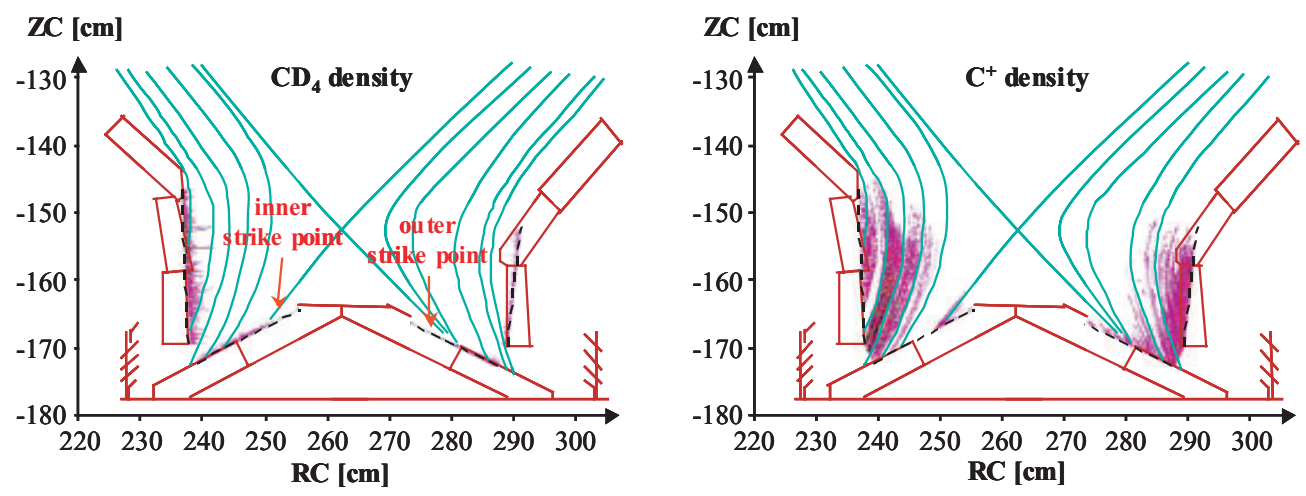

Figure 2. Simulated $\mathrm{CD}_{4}$ (left) and $\mathrm{C}^{+}$(right) particle density after chemical erosion of $\mathrm{CD}_{4}$ in the divertor of JET MkIIa under the assumption of fully sticking for hydrocarbons.

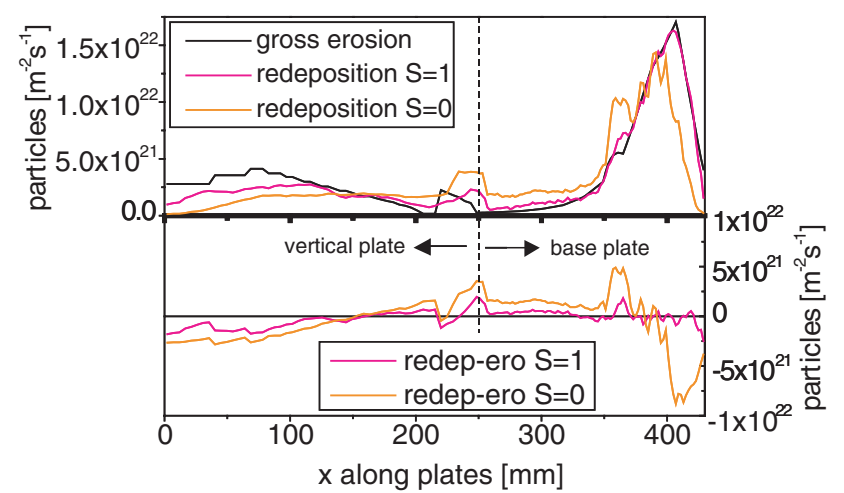

Figure 3. Profiles of erosion and re-deposition along the inner divertor plates for fully $(S=1)$ and zero $(S=0)$ sticking of hydrocarbons (upper part). Resulting profiles of re-deposition minus erosion (lower part). 
the sticking probability of hydrocarbon fragments returning to the divertor plates the extreme cases of fully $(S=1)$ and zero-sticking $(S=0)$ have been analysed. In the latter case the hydrocarbons are re-ejected as saturated methane molecules into the edge plasma. A negligible sticking of hydrocarbons cannot be explained solely with a simple energetic reflection but with a high (self) re-erosion of a soft a-C : D layer built up by the hydrocarbons [13]. At first, the sticking of carbon atoms and ions has been determined using reflection coefficients calculated with the TRIM code [14].

To visualize the local transport of eroded methane molecules figure 2 shows the twodimensional distribution of the $\mathrm{CD}_{4}$ and $\mathrm{C}^{+}$density for the assumption of sticking $S=1$ of hydrocarbons. Due to the high electron density near to the strike points the penetration depth of $\mathrm{CD}_{4}$ is extremely small in these regions $(\sim \mathrm{mm})$. At locations away from the strike points the penetration depth is in the order of a cm or slightly smaller. The $\mathrm{C}^{+}$ions, which are a reaction product at the end of the dissociation chain of $\mathrm{CD}_{4}$ molecules, penetrate deeper into the plasma $(\sim 10 \mathrm{~cm})$. Coulomb interactions with the background plasma ions (friction force) tend to drive the ions back to the divertor plates or into the louver regions via gyration around the magnetic field lines (figure 2, right-hand side). The density distributions of the different species for the assumption of zero-sticking $S=0$ for hydrocarbons do not differ significantly from the case of fully sticking $S=1$. Nevertheless, the different sticking assumptions lead to differences in the re-deposition such that the integrated amount of redeposition relative to the amount of eroded molecules decreases in the case of zero-sticking. At the inner divertor re-deposition fractions vary from $97 \%(S=1)$ to $86 \%(S=0)$. A similar behaviour is found at the outer divertor: the re-deposition decreases from $98 \%$ to $85 \%$. The profiles of erosion and re-deposition along the plates are shown in figure 3 for the inner divertor, upper part. Only the inner divertor is shown since the simulations do not reveal significant differences between inner and outer divertor. The co-ordinate $x$ used in figure 3 is the position along the plates with the starting point $x=0$ corresponding to the upper corner of the vertical plate $(\mathrm{ZC}=-148 \mathrm{~cm})$ and the end point $x=430 \mathrm{~mm}$ to the right end of the horizontal (base) plate $(\mathrm{RC}=255 \mathrm{~cm})$. For the case of fully sticking $(S=1)$ almost all particles eroded near to the strike point $(x \approx 400 \mathrm{~mm})$ are re-deposited locally. This is a consequence of the above-mentioned short penetration of $\mathrm{CD}_{4}$ molecules in this region. Particles eroded at the vertical plates have larger penetration depths and therefore a higher probability to be transported away from their origin. Thus at low $x$-values on the vertical plates the re-deposition probability is small and part of the eroded particles are transported downwards along the vertical plates and are re-deposited (at higher $x$-values) or enter the louver region. Under the assumption of zero-sticking the re-deposition profile near to the strike point is significantly shifted along the horizontal plates towards the louver region (to smaller $x$-values). Most of the eroded molecules return to the plate near to their erosion site as hydrocarbon fragments. Due to the assumption $S=0$ they are re-ejected into the plasma. This process is repeated until the particle is re-deposited as a carbon atom or ion with a re-deposition probability according to TRIM which is significantly high under the given plasma conditions (small reflection coefficient). The repetition of these processes and the movement of the charged species along the magnetic field lines towards the plate leads to a deposition left from the erosion site deeper in the SOL (smaller $x$-values). In the lower part of figure 3 the resulting net-deposition and net-erosion patterns along the plates for the two sticking assumptions are shown.

Table 1 summarizes the integrated amount of re-deposition at the inner and outer divertor for the different sticking assumptions. The amounts of particles entering the louver regions show no significant difference for the inner and outer divertor. This is in strong contradiction to the observations showing large carbon deposition at the inner louvers and almost nothing 
Table 1. Integrated amount of re-deposition and particles entering the louver regions for fully and zero-sticking of hydrocarbons in the inner and outer divertor.

\begin{tabular}{|c|c|c|c|c|}
\hline & \multicolumn{2}{|c|}{ Inner divertor } & \multicolumn{2}{|c|}{ Outer divertor } \\
\hline & $\begin{array}{l}\text { Re-deposition } \\
(\%)\end{array}$ & $\begin{array}{l}\text { Particles to } \\
\text { louver (\%) }\end{array}$ & $\begin{array}{l}\text { Re-deposition } \\
(\%)\end{array}$ & $\begin{array}{l}\text { Particles to } \\
\text { louver (\%) }\end{array}$ \\
\hline$S=1$ & 97 & 1.5 & 98 & 1 \\
\hline$S=0$ & 86 & 10 & 85 & 12 \\
\hline
\end{tabular}

at the outer. Evidently, additional processes determine the transport of eroded carbon. One candidate is an asymmetric flow driving particles which are eroded at the main chamber, and maybe also at the outer vertical tile, to the inner divertor. This would result in an essentially zero carbon background flux to the outer divertor but a large background contribution to the inner. Measurements of the Mach number at JET indicate such flows even though there is no final explanation of their origin [7]. In the outer divertor we have thus a situation with a low fraction of eroded carbon reaching the louver as simulated with the code leading to no significant carbon deposition. But the code does not reproduce the measured amount of carbon that is deposited at the inner louvers. This amount has been estimated experimentally to be $\approx 4 \%$ of the incoming deuterium ion fluence $\Phi_{\mathrm{D}+}$ to the inner divertor region [4]. The amounts simulated are $\approx 0.08 \% \cdot \Phi_{\mathrm{D}+}$ for $S=1$ and $\approx 0.5 \% \cdot \Phi_{\mathrm{D}+}$ for $S=0$ assuming a chemical erosion yield of $Y_{\mathrm{D}+\rightarrow \mathrm{CD}_{4}}=5 \%$. Thus even for $S=0$ the simulated value is at least one order of magnitude too small keeping also in mind that only some fraction of particles entering the louver region is finally deposited at the louvers.

Thus, for the inner divertor we will discuss the possible influence of further parameter variations while for the sticking probability of hydrocarbons only zero-sticking is considered.

2.1.1. Dynamic reflection of carbon atoms and ions. TRIM calculations of the reflection of carbon on graphite predict zero reflection for particle energies less than $\approx 20 \mathrm{eV}$. At these low energies chemical and collective processes become more and more important such that the binary collision model used in TRIM becomes more and more invalid. In contrast, calculations with the molecular dynamics code MolDyn [15] result in a significant reflection of carbon particles on an a-C : D layer as shown in figure 4 for an impact angle of $60^{\circ}$. Using these reflection coefficients instead of those of TRIM decreases the simulated amount of re-deposition for the inner divertor from $86 \%$ to $75 \%$. At the same time the amount of particles entering the louver region (relative to the amount of eroded particles) increases from $9 \%$ to $12 \%$. Although this increase tends to the right direction the absolute value of $12 \%$ is still about one order of magnitude too small (if a chemical erosion $Y_{\mathrm{D}+\rightarrow \mathrm{CD}_{4}}=5 \%$ is assumed) in order to explain the measurements.

2.1.2. New rate coefficients for hydrocarbons. Recently new data for the rate coefficients for electron and proton reactions of hydrocarbons were published [16,17]. They differ significantly from the Ehrhardt-Langer database used so far-details are discussed elsewhere [18]. Additional ERO-JET simulations have been done with the rate coefficients for electron reactions of [16] and with proton reactions of [17]. This leads to a further decrease of the re-deposition along the inner divertor plates from $75 \%$ to $69 \%$ while the simulated amount of particles towards the inner louver region increases from $12 \%$ to about $15 \%$. 


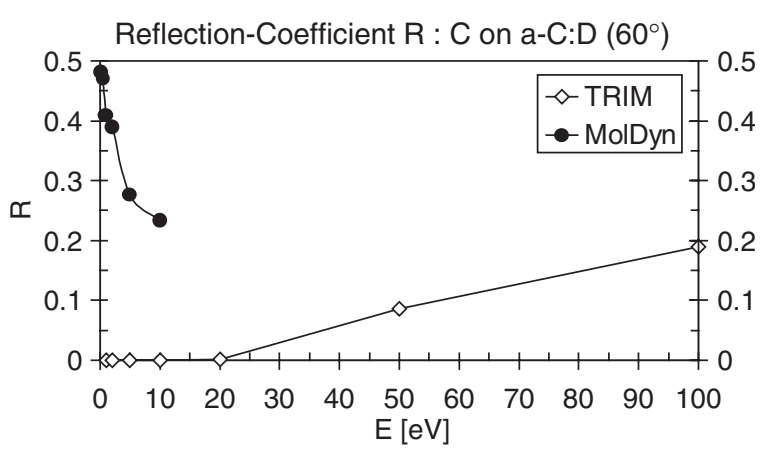

Figure 4. Reflection coefficient for 'carbon on carbon' according to MolDyn [15] calculations in comparison to TRIM [14].

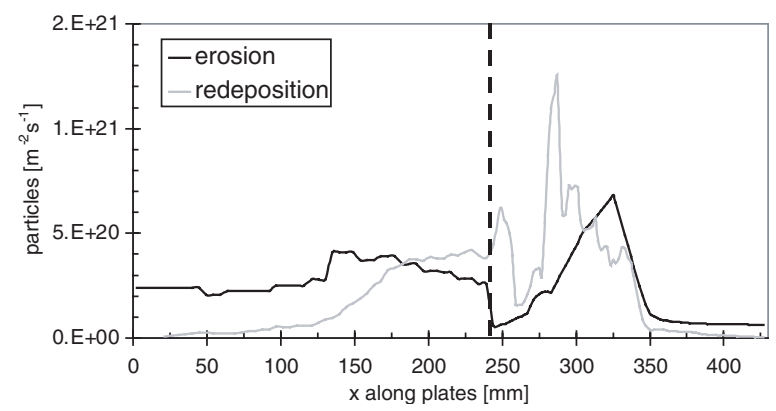

Figure 5. Profiles of erosion and re-deposition along the inner divertor plates using zero-sticking for hydrocarbons, MolDyn reflection coefficients for carbon atoms/ions and the plasma with the shifted strike point.

2.1.3. Location of the strike point. In the Onion Skin Modelling of the inner divertor the strike point at the horizontal plate is located at $x \approx 400 \mathrm{~mm}$ (see figure 3, maximum of erosion). Several measurements, however, indicate a shift of the strike point at the horizontal plates by $\approx 8 \mathrm{~cm}$ to the high field, left side [4]. Thus ERO-JET calculations were carried out for the inner divertor with an OSM plasma shifted as a whole by $8 \mathrm{~cm}$ to the left side along the horizontal plate. The resulting profiles of erosion and re-deposition (Ehrhardt-Langer data, zero-sticking for hydrocarbons, dynamic reflection of carbon particles) are shown in figure 5. Compared to the 'non-shifted plasma' the re-deposition increases from $75 \%$ to $82 \%$, resulting from an increase of the average temperature and density of the shifted plasma. The amount of particles entering the louver region increases also somewhat, from $12 \%$ to $15.3 \%$, since more particles are eroded at locations closer to the louver region.

2.1.4. Electron temperature and density variations. Due to uncertainties in using the OSM plasma parameters as representative for the 10000 s of plasma operation in JET MkIIa the influence of varying plasma conditions on the particle transport is investigated. $T_{\mathrm{e}}$ and $n_{\mathrm{e}}$ have been varied by a factor of 2 keeping the plasma pressure $T_{\mathrm{e}} n_{\mathrm{e}}$ constant while the spatial distribution according to the OSM solution was kept unchanged. This procedure changes the incoming ion flux density $\left(\sim n_{\mathrm{e}} T_{\mathrm{e}}^{0.5}\right)$. The results are summarized in figure 6 . While the re-deposition decreases only slightly with increased incoming deuterium ion flux the amount of particles to the louvers decreases by more than a factor of 2 (from $16 \%$ to $6.3 \%$ ). This is 


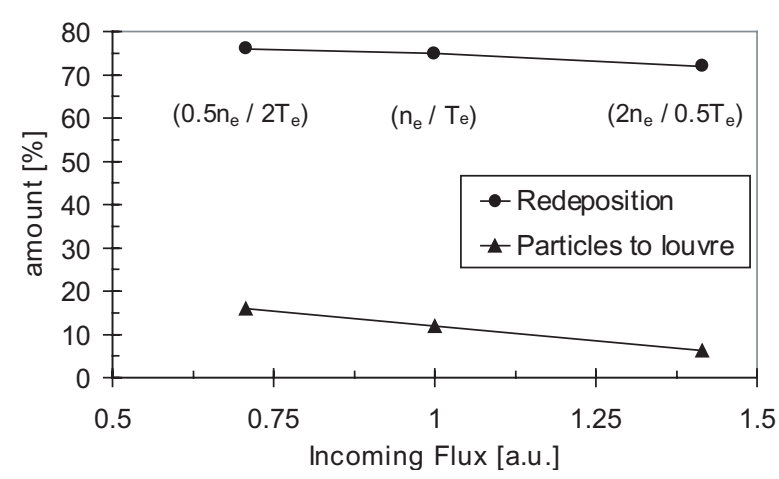

Figure 6. Influence of variations of the electron temperature and density on the integrated re-deposition and on the amount of particles entering the louver region in the inner divertor for chemically eroded $\mathrm{CD}_{4}$.

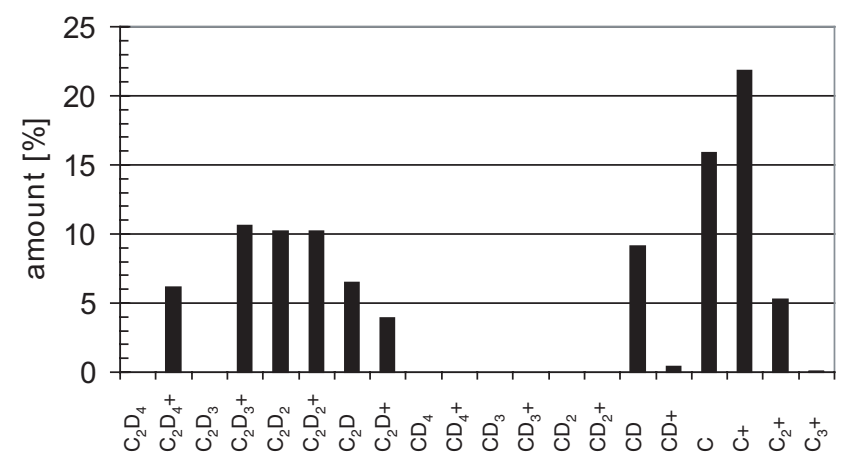

Figure 7. Simulated distribution of species returning to the inner divertor plates after chemical erosion of $\mathrm{C}_{2} \mathrm{D}_{4}$.

attributed to an increased number of particles leaving the simulation volume in positive $r$ - and $z$-direction as a consequence of the decreased electron temperature and is thus also partly an effect of the assumed size of the simulation volume.

\subsection{Contribution of higher hydrocarbons: $C_{2} D_{4}$}

At low electron temperatures - as present in the divertor regions-the formation of higher hydrocarbons $C_{2} D_{x}$ and $C_{3} D_{x}$ becomes more and more important [19]. To estimate its importance simulations with chemically eroded $\mathrm{C}_{2} \mathrm{D}_{4}$ (which is one of the most important species at low temperatures, [19]) were carried out. Rate coefficients for electron and proton reactions with $\mathrm{C}_{2} \mathrm{D}_{4}$ are taken from $[16,17]$. With the 'non-shifted' plasma solution, the zerosticking assumption for hydrocarbons and dynamic reflection of carbon atoms and ions the resulting re-deposition profile is similar to the one for methane molecules (figure 3). The fraction of re-deposition is slightly smaller than the one for $\mathrm{CD}_{4}$ erosion $(69 \%$ compared to $75 \%$ ). Figure 7 shows the distribution of species returning to the divertor plates from the $\mathrm{C}_{2} \mathrm{D}_{4}$ molecule (first cycle, re-ejected hydrocarbons are not included). Under those conditions, about $39 \%$ of the eroded $\mathrm{C}_{2} \mathrm{D}_{4}$ returns to the plates as (charged or neutral) $\mathrm{C}_{2} \mathrm{D}_{x}, 8 \%$ as $\mathrm{CD}$ and $37 \%$ as carbon atom or ion. Figure 8 presents the integrated re-deposition for the $\mathrm{C}_{2} \mathrm{D}_{4}$ molecules together with the amount of particles reaching the louver region. The value of one for the normalized flux corresponds to the plasma conditions from the Onion Skin Model 


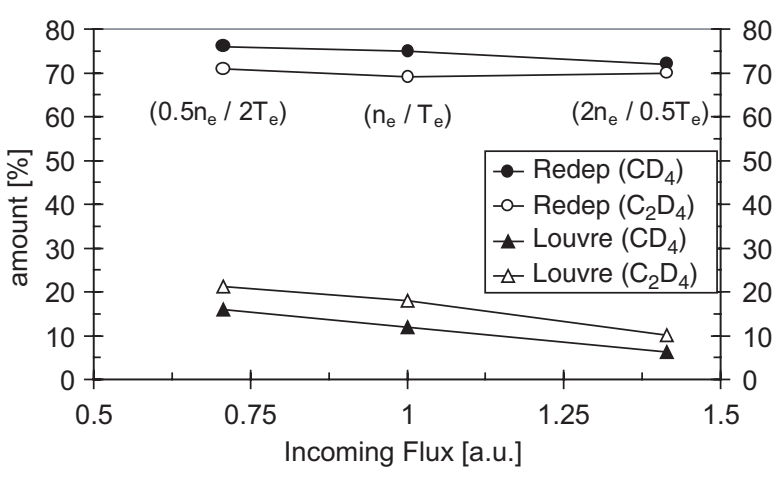

Figure 8. Influence of variations of the electron temperature and density on the integrated re-deposition and on the amount of particles entering the louver region in the inner divertor for chemical erosion of $\mathrm{C}_{2} \mathrm{D}_{4}$ and $\mathrm{CD}_{4}$.

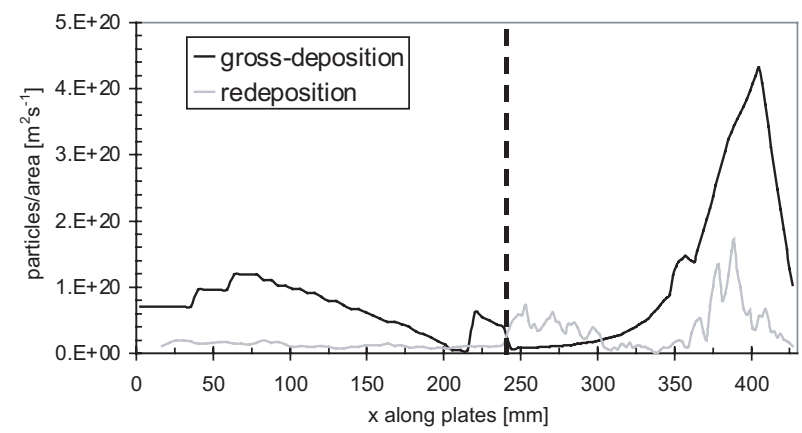

Figure 9. Simulated carbon deposition at the inner divertor plates resulting from the incoming background plasma.

calculations. Figure 8 also includes the results for $\mathrm{CD}_{4}$. As for $\mathrm{CD}_{4}$ the re-deposition of $\mathrm{C}_{2} \mathrm{D}_{4}$ shows no clear dependence on the incoming flux but the values themselves are slightly smaller than those for $\mathrm{CD}_{4}$. The amount of particles entering the louver decreases with increasing flux and is higher for $\mathrm{C}_{2} \mathrm{D}_{4}$ than for $\mathrm{CD}_{4}$.

\subsection{Background carbon flux}

The deposition of carbon from the background carbon flux is very important for erosion and deposition at the divertor plates. The assumption of a certain fraction of carbon flux relative to the incoming deuterium ion flux results in a deposition profile shown in figure 9. The grossdeposition is here the amount of incoming background carbon which is directly deposited. With the reflection coefficients from the dynamic MolDyn calculations and the 'non-shifted' plasma solution, $67 \%$ of the incoming carbon flux is gross-deposited and $33 \%$ is reflected. $52 \%$ of the reflected carbon is finally re-deposited so that, altogether, around $84 \%$ of the incoming background carbon is deposited with a maximum of deposition near the strike point. Carbon particles, which are not deposited enter the louver region or leave the calculation volume. Table 2 summarizes the amount of gross-reflection (incoming background carbon which is directly reflected), re-deposition and particles entering the louver region for TRIM reflection coefficients instead of MolDyn and the shifted plasma solution. Compared to MolDyn data the TRIM reflection coefficients lead to a significant decrease of the gross-reflection from $33 \%$ to 
Table 2. Gross-reflection and re-deposition of the incoming background carbon: reflection coefficients according to TRIM compared to MolDyn.

\begin{tabular}{llll}
\hline & TRIM & \multicolumn{2}{c}{ Dynamic reflection } \\
\cline { 2 - 4 } & $\begin{array}{l}\text { Non-shifted } \\
\text { plasma (\%) }\end{array}$ & $\begin{array}{l}\text { Non-shifted } \\
\text { plasma (\%) }\end{array}$ & $\begin{array}{l}\text { Shifted } \\
\text { plasma (\%) }\end{array}$ \\
\hline 'Gross' reflection (relative to incoming background carbon) & 3.6 & 33 & 33 \\
Re-deposition (relative to 'gross' reflected carbon) & 74 & 52 & 67 \\
Particles to louver (relative to incoming background carbon) & 0.4 & 4 & 6 \\
\hline
\end{tabular}

less than $4 \%$. The amount of particles, which can enter the louver region decreases by a factor of ten from $4 \%$ to $0.4 \%$. The influence of the plasma with the shifted strike point is comparably small: with the MolDyn data the amount of gross-reflected particles does not change whereas the integrated amount of re-deposition increases slightly from 52\% to $67 \%$ and the particles entering the louver region from $4 \%$ to $6 \%$.

\section{Comparison to experimental observations and discussion of soft film formation and erosion}

The following discussion concentrates on the carbon deposition at the inner louvers whereas the in-out asymmetry of the deposition, which seems to be a global transport effect caused by asymmetric flows, is not considered. Experimentally, the amount of carbon particles contributing to the deposition at the inner louvers was estimated to $\sim 4 \%$ of the incoming deuterium ion fluence $\Phi_{\mathrm{D}+}$ [4]. This is an integrated result of about 10000 s plasma operation in JET MkIIa. With the plasma parameters from the Onion Skin Model calculations, zerosticking for hydrocarbons and the dynamically calculated reflection coefficients for carbon atoms and ions the ERO-JET calculations result in an amount of carbon of $0.52 \% \cdot \Phi_{\mathrm{D}+}$ entering the louver region with the following contributions:

- chemical erosion of $\mathrm{CD}_{4}$ with $Y_{\mathrm{D}+\rightarrow \mathrm{CD}_{4}}=1 \% \Rightarrow 0.12 \% \cdot \Phi_{\mathrm{D}+}$,

- chemical erosion of $\mathrm{C}_{2} \mathrm{D}_{4}$ with $Y_{\mathrm{D}+\rightarrow \mathrm{C}_{2} \mathrm{D}_{4}}=1 \% \Rightarrow 0.36 \% \cdot \Phi_{\mathrm{D}+}$,

- background carbon flux with $\Gamma_{\mathrm{C}}=1 \% \Rightarrow 0.04 \% \cdot \Phi_{\mathrm{D}+}$.

The value of $0.52 \%$ is a factor of 8 smaller than measured. However, the above values of $1 \%$ for chemical erosion caused by deuterium ions and $1 \%$ background carbon flux are rough assumptions. Indeed, measurements at JET suggest higher values, that is $5 \%$ for $\mathrm{CD}_{4}$ erosion, $7 \%$ for $\mathrm{C}_{2} \mathrm{D}_{4}$ erosion [20] and $5 \%$ for $\Gamma_{\mathrm{C}}$ [21]. With these values the ERO-JET simulations result in an amount of $3.3 \% \cdot \Phi_{\mathrm{D}+}$ carbon particles entering the louver region, which is very near to the measured value of $4 \%$. A further increase (to $4.6 \%$ ) is achieved if the shiftedstrike point is taken into account. Nevertheless, the involved overall chemical erosion of $19 \%$ related to the amount of eroded carbon particles seems to be unlikely-at least as a stationary value during the whole campaign. This would automatically lead to net erosion zones near the strike point unless the background carbon flux is high enough to compensate the erosion, thus about $20 \%$. However, the inner divertor plates are deposition dominated [4-6]. Therefore 'standard' modelling as described above cannot reproduce the experimental observations and a better set of input data is required. In any case, the considered input parameter variations (temperature, density, strike point position and sticking of hydrocarbons) are not sufficient to resolve the disagreement. Additional mechanisms are therefore one further possibility - two are discussed in the following: first, the chemical erosion of soft (re)-deposited carbon-films can be significantly higher than that of pure graphite [22]. Second, 
the chemical erosion due to deuterium atoms is not included so far in the simulations but could have an important contribution in addition to deuterium ions [22]. Our present view to explain the experimental observations is as follows: carbon is eroded at the first wall and (partly) at the outer divertor (these are erosion dominated areas in JET [4-6]) and transported-via asymmetric flows - into the inner divertor [4,7]. The main part (around $80 \%$, see chapter 2.3) of the incoming carbon is deposited at the divertor plates in form of soft, hydrogen-rich films. These soft films are re-eroded effectively by chemical erosion-at locations with low deuterium ion fluxes away from the strike point this erosion could take place via deuterium atoms. Most of the eroded carbon (around $80 \%$, see chapter 2) is then first of all re-deposited at the divertor plates. As a result of the transport of charged species along the magnetic field lines the location of re-deposition is shifted relative to the location of erosion into the direction of the louver region as suggested by the simulations. The repetition of these processes (erosion of re-deposited layers - transport along magnetic field linesre-deposition) finally leads to a successive transport of the carbon particles into the inner louver until a net-deposition of carbon takes place at the water-cooled louvers. Also, the temperature of the substrate is certainly an important parameter. At the divertor plates with temperatures around $200^{\circ} \mathrm{C}$ the soft carbon films are chemically eroded effectively whereas at the water-cooled louvers (surface temperature around $20^{\circ} \mathrm{C}$ ) the chemical erosion yield is about an order of magnitude reduced which can lead to a significant net-deposition at these locations [22].

\section{Conclusions and conjectures}

Simulations of the carbon transport in the divertor of JET MkIIa cannot reproduce the huge carbon deposition at the inner louvers if 'standard' assumptions (1\% erosion yield due to erosion via deuterium ions, such yields based in part on data) are used. However, the simulations suggest that a particle transport in the inner divertor dominated by re-deposition and further effective erosion of soft carbon layers finally can lead to a successive transport of carbon to the louver region. In addition to chemical erosion due to deuterium ions the erosion due to deuterium atoms seems to be important so that even at locations with low $\mathrm{D}^{+}$-fluxes (away from the strike point) an effective erosion of the soft re-deposited carbon layers can take place. As a result the main part of the background carbon, which enters the inner divertor is successively transported into the louver region via (re)-deposition and erosion and leads to thick carbon layers at the inner water-cooled louvers. This model is, however, somewhat speculative and needs further confirmation.

In this picture the carbon arriving at the water cold louvers is from the main wall. Based on this, the expected carbon deposition behaviour in ITER, where only the divertor plates are made from CFC with no main wall as a carbon source, should be largely different: the possible source of carbon particles, which can lead to the formation of soft films under special plasma conditions is eliminated and the formation of tritium containing carbon layers via co-deposition should be less problematic in ITER. In addition, the deposition of beryllium from the main chamber at the divertor plates may lead to a significant decrease of carbon erosion of the target plates itself. This will most probably reduce the quantity of T-retention in ITER if compared with numbers extrapolated from machines with full carbon walls. However, in order to fulfil the safety limits a very strong reduction factor has to be achieved which we will not suggest to be achieved with these considerations here. Indeed, much more experimental and modelling work is necessary to consolidate predictions of the long term T-retention in ITER significantly. 


\section{Acknowledgments}

The authors would like to thank P Stangeby and D Elder for providing modelling calculations of the background plasma in the MkIIa divertor of JET and W Fundamenski for fruitful discussions. US work was supported by the US Department of Energy.

\section{References}

[1] ITER Technical Basis 2002 ITER EDA Documentation Series No 24 (Vienna: IAEA)

[2] Janeschitz G, ITER JCT and HTs $2001 \mathrm{~J}$. Nucl. Mater. 290-293 1

[3] Vlases G C et al 1999 J. Nucl. Mater. 266-269 160

[4] Coad J P et al 2001 J. Nucl. Mater. 290-293 224

[5] Whyte D G, Coad J P, Franzen P and Maier H 1999 Nucl. Fusion 391025

[6] Coad J P et al 2002 Erosion/deposition in JET during the period 1999-2001 Contribution to the 15th PSI Conf. (Gifu, May 2002)

[7] Erents S K et al 1999 Proc. 26th EPS Conf. on Controlled Fusion and Plasma Physics (Maastricht, June 1999)

[8] Likonen J et al 2002 Studies of impurity deposition/implantation in JET divertor tiles using SIMS and ion beam techniques Contribution to the 22nd Symp. on Fusion Technology (Helsinki, September 2002)

[9] Kirschner A, Philipps V, Winter J and Kögler U 2000 Nucl. Fusion 40989

[10] Fundamenski W, Stangeby P C and Elder J D 1999 J. Nucl. Mater. 266-269 1045

[11] Fundamenski W 1997 J. Nucl. Mater. 290-293 593

[12] Stangeby P C et al 1997 J. Nucl. Mater. 241-243 358

[13] Wienhold P et al 2001 J. Nucl. Mater. 290-293 362

[14] Eckstein W 1991 Computer Simulation of Ion Solid Interaction (Berlin: Springer)

[15] Alman D A, Ruzic D N and Brooks J N 2002 Molecular dynamics calculations of carbon/hydrocarbon reflection coefficients on a graphite surface employing distributed computing Contribution to the 15th PSI Conf. (Gifu, May 2002)

[16] Brooks J N, Wang Z, Ruzic D N and Alman D A 1999 ANL/FPP/TM-297

[17] Janev R K, Wang J G and Kato T 2001 NIFS-DATA-64

[18] Kirschner A et al 2002 Contribution to the 15th PSI Conf. (Gifu, May 2002)

[19] Mech B V, Haasz A A and Davis J W 1997 J. Nucl. Mater. 241-243 1147

[20] Stamp M F et al 2001 J. Nucl. Mater. 290-293 321

[21] McCracken G M et al 1999 Nucl. Fusion 3941

[22] Vietzke E and Haasz A A 1996 Physical Processes of the Interaction of Fusion Plasmas with Solids ed W O Hofer and J Roth (San Diego: Academic) 